\title{
Integriertes Präkonzentrator-Gassensor-Mikrosystem zur Detektion von Spurengasen in Innenraumluft
}

\author{
Martin Leidinger ${ }^{1}$,Max Rieger ${ }^{2}$, Tilman Sauerwald ${ }^{1}$, Christine Alépée ${ }^{3}$, Andreas Schütze ${ }^{1}$ \\ ${ }^{1}$ Universität des Saarlandes, Lehrstuhl für Messtechnik, Campus A5 1, 66123 Saarbrücken, \\ Deutschland \\ ${ }^{2}$ Fraunhofer Institut für Chemische Technologie ICT, Joseph-von-Fraunhoferstraße 7, 76327 Pfinztal, \\ Deutschland \\ ${ }^{3}$ SGX Sensortech SA, Courtils 1, 2035 Corcelles / NE, Schweiz
}

\begin{abstract}
Zusammenfassung
Die Funktionsweise eines integrierten Mikrosystems von Präkonzentratoren und Gassensoren wird. mit Hilfe der Finiten Elemente Methode (FEM) untersucht. Als Präkonzentratormaterial wird MIL-53, ein spezielles Metallorganisches Netzwerk (metal organic framework,MOF) verwendet. Mit Hilfe des Modells lässt sich basierend auf dem temperaturabhängigen Verteilkoeffizient und den Diffusionseigenschaften ein Faktor für die Aufkonzentration über die Zeit bestimmen. Der Verteilkoeffizient zwischen Präkonzentrator und Luft wurde mittels inverser Gaschromatographie (iGC) bestimmt. Für eine Benzolkonzentration von $10 \mathrm{ppb}$ in Luft bei einer Adsorptionstemperatur von $50^{\circ} \mathrm{C}$ für $300 \mathrm{~s}$ und einer Desorptionstemperatur von $200^{\circ} \mathrm{C}$ wird am Ort der Sensoren eine Konzentrationsspitze von fast $250 \mathrm{ppb}$ vorhergesagt. Für Toluol ist unter den gleichen Bedingungen die Aufkonzentration deutlich geringer (ca. $160 \mathrm{ppb}$ ). Testmessungen zeigen eine erfolgreiche Anwendung des Prinzips, allerdings ist dies auf niedrige relative Feuchten begrenzt. Bei hohen relativen Feuchten treten Effekte auf, die zu einer starken Abnahme der Sorptionseigenschaften führen.
\end{abstract}

Keywords: Gassensorik, Präkonzentrator, Metal-organic frameworks, VOC-Messung, Indoor Air Quality.

\section{Einleitung}

Die Luftqualität in Innenräumen kann durch gasförmige Komponenten, besonders flüchtige organische Verbindungen (Volatile organic compounds, VOCs), beeinträchtigt werden. Neben Messung der Summenkonzentration an VOC rückt die Messung einzelner besonders giftiger Komponenten zunehmend in das Interesse [1,2]. Einige dieser Substanzen können bereits in sehr geringen Konzentrationen im unteren ppb-Bereich Gesundheitsprobleme hervorrufen. Eine besondere Herausforderung ist die Messung von Benzol, welches aufgrund seiner krebserregenden Eigenschaften einen sehr niedrigen Akzeptanzwert von 1,6 ppb hat [3]. In früheren Arbeiten haben wir gezeigt, dass z.B. mittels Metalloxid-Halbleiter-Gassensoren im temperaturzyklischen Betrieb eine solche Messung im Labor auch selektiv zu anderen VOCs möglich ist [4]. Um auch unter stark schwankender Konzentration von Störgasen, wie sie in realen Umgebungsbedingungen $\mathrm{zu}$ erwarten ist, eine sichere Benzolmessung zu gewährleisten, ist eine spezifische Verstärkung des Benzolsignals erforderlich Dies kann z.B. durch eine Aufkonzentration des Gases erzielt werden. Mit geeigneten Materialien mit hoher Oberfläche können Gasteilchen über einen Zeitraum gesammelt und dann mittels thermischer Desorption schnell wieder abgegeben werden, wodurch ein kurzer Puls hoher Gaskonzentration entsteht. Mit dieser Methode lässt sich der Einfluss von Permanentgasen (z.B. $\mathrm{CO}$ und $\mathrm{H}_{2}$ ) auf das Sensorsignal verringern. Über eine geeignete Wahl der Adsorptions- und Desorptionstemperatur lassen sich Dämpfe mit einer spezifischen Flüchtigkeit gezielt anreichern [5]. Als besonders leistungsfähige Materialien für Präkonzentratoren $(\mathrm{PC})$ haben sich in jüngeren Arbeiten Metallorganische Gerüstverbindungen (Metal-organic frameworks, MOFs) erwiesen [6] z.B. MIL-53 [7]. Diese Verbindungen besitzen eine sehr hohe spezifische Oberfläche (bis zu über $1000 \mathrm{~m}^{2} / \mathrm{g}$ ), wodurch große Mengen an Gasmolekülen in einem kleinen Volumen gebunden werden können. Im Vergleich zu Zeolithen können die chemischen 
Eigenschaften der Poren stärker variiert werden, was besonders für vorwiegend unpolare Gase wie die VOCs von Vorteil ist. MOFs bestehen aus Metall- oder MetallCluster-Kationen („Knoten“) und organischen Linker-Molekülen [6]. In früheren Untersuchungen konnten wir zeigen, dass ein spezifisches MOF, MiL-53, deutlich bessere Eigenschaften aufweist als das weit verbreitete Tenax $^{\circledR}$ TA. Dies gilt insbesondere für kleine Konzentrationen von leichtflüchtigen Substanzen wie z.B. Benzol. Das Retentionsvolumen und damit der Verteilkoeffizient zwischen dem Adsorbens und Luft ist für MIL-53 um zwei Größenordnungen größer als für Tenax $^{\circledR}$ (Abb. 1). Es lagert sich im Gleichgewicht also erheblich mehr Benzol im Material an. Außerdem variiert der Verteilkoeffizient stärker mit der Temperatur (was nach dem van t'Hoff Gesetz direkt aus der höheren Bindungsenergie resultiert). Die Bestimmung der Sorptionseigenschaften bezüglich der Zielgase (Benzol, Toluol) erfolgte mittels inverser Gaschromatographie an einem herkömmlichen Gaschromatographen mit
Photoionisationsdetektor. Zu diesem Zweck wurde eine MOF gepackte Säule zwischen Injektor und Detektor eingebracht. Die Durchbruchvolumina wurden mittels eines Masseflussmessers und der Retentionszeit unter Berücksichtigung der Totzeit (Methan) bestimmt. Eine detaillierte Beschreibung des Aufbaus und ein Vergleich mit anderen MOF sowie herkömmlichen Adsorbentien (Tenax ${ }^{\circledR}$ TA) findet sich in [5]. In dieser Arbeit untersuchen wir die Verwendung von MIL-53 als Präkonzentrator für Benzol in einem integrierten Mikrosystem. Dazu wurde ein integriertes Präkonzentrator-GassensorSystem entwickelt, in dem das MOF-Material auf einer Micro-Hotplate zusammen mit einem Gassensorchip in einem Gehäuse integriert wurde. Die physikalischen Vorgänge in diesem System bezüglich der Gasadsorption und desorption wurden in FEM-Simulationen näher untersucht. Die integrierten Systeme wurden schließlich auf ihre Benzol-Detektionsleistung bei verschiedenen Umgebungsbedingungen getestet, insbesondere bei wechselnder Gasfeuchte.

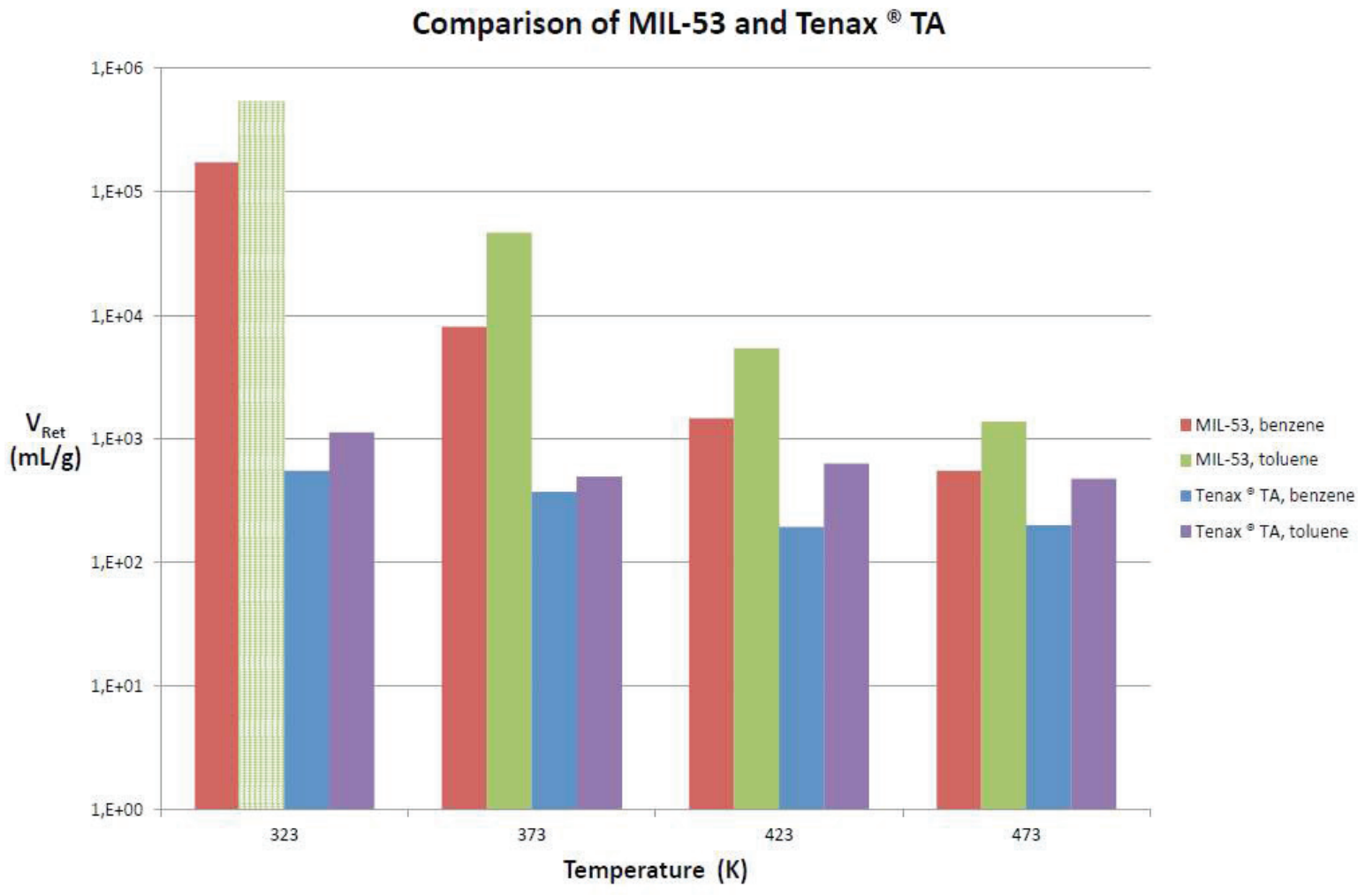

Abb. 1: Vergleich der Retentionsvolumina von MIL-53 und Tenax $\AA$ TA bei verschiedenen
Temperaturen 


\section{MOF-Präkonzentratormaterialien für VOCs}

Für die Beschichtung der PräkonzentratorHotplate wird MIL53 Pulver (Sigma Aldrich, Handelsname Basolite A100) verwendet. Die Partikelgröße wurde mit Hilfe dynamischer Lichtstreuung zu $19.8 \mu \mathrm{m}$ bestimmt.

Die Funktionsweise der Präkonzentratorschicht wurde mittels Massenspektrometrie getestet. Zu diesem Zweck wurde MOF-Pulver in di-nButyläther suspendiert und ca. $30 \mathrm{mg} \mathrm{MOF}$ wurden als Suspension auf eine $3 \times 3 \mathrm{~mm}^{2}$ große Hotplate aus Alumina aufgebracht. Diese Hotplate (UST Umweltsensortechnik $\mathrm{GmbH}$, Geschwenda) verfügt über einen integrierten Platinheizer, der mit Hilfe einer Elektronikplattform (Toolbox, $3 \mathrm{~S} \mathrm{GmbH}$, Saabrücken) temperaturgeregelt betrieben wird. Das System wurde in einem konstanten Massestrom getestet. Die Gasproben wurden mit einem Gasprobenbeutel (Supelco, 30240$\mathrm{U})$ bereitgestellt. Der Ausgangsgasstrom wurde mit Hilfe eines Massenspektrometers (Pfeiffer GSD 301 Quadrupol-MS) überwacht. Nach variablen Anreicherungszeiten wurde der Präkonzentrator ausgeheizt (für $500 \mathrm{~s}$ auf $200^{\circ} \mathrm{C}$ ) Für Zeiten unterhalb von 20 min wurde ein linearer Anstieg des Desorptionspeaks beobachtet während für längere Zeitintervalle eine deutliche Abflachung der Kurven zu beobachten ist [5], was auf eine Sättigung des Präkonzentrators hindeutet.

\section{Integriertes Präkonzentrator-Sensorsystem}

Das MOF-Material wurde auf einer MicroHotplate aufgetragen und diese zusammen mit einem Dual-MEMS-Gassensor-Chip (SGX Sensortech SA) in einem Keramikgehäuse $\left(7 \times 5 \times 1 \mathrm{~mm}^{3}\right)$ integriert. Das gesamte System bildet ein SMD-Bauteil („surface mounted device") mit zehn elektrischen Kontakten.

Die Geometrie des Aufbaus wurde in COMSOL Multiphysics implementiert, um die Vorgänge innerhalb des Systems näher untersuchen zu können, siehe Abb. 2. Die beiden Chips sind nebeneinander angeordnet, mit ca. 0,5 mm Abstand zum Deckel. Auf der Seite des Präkonzentrators ist ein Gaseinlass mit einer Breite von 1,5 mm realisiert, das Gas aus der Umgebung kann nur über diese Öffnung in das System gelangen. Dies führt zum einen dazu, dass der Präkonzentrator Gas aus der Umgebung aufnehmen kann, zum Anderen adsorbiert so das Gas aus dem Gehäuse im MOF, die Gaskonzentration an den Sensoren kann damit vorübergehend deutlich unter den Gleichgewichtswert verringert werden.

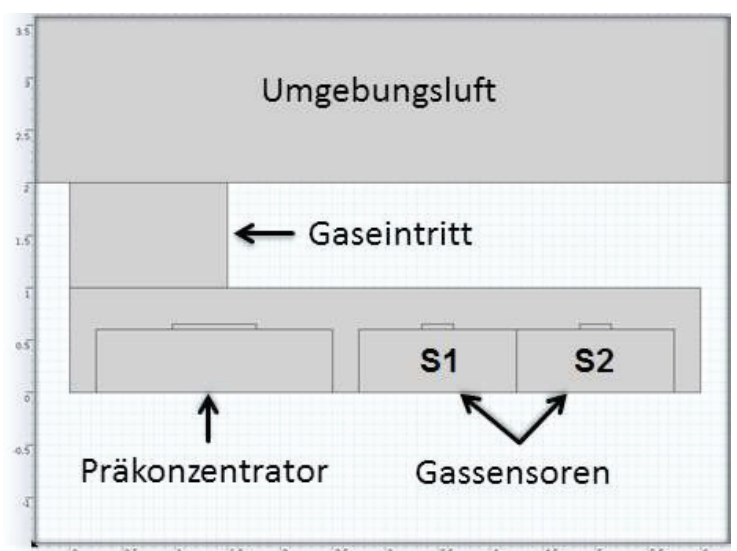

Abb. 2: Geometrischer Aufbau des integrierten Systems in COMSOL, nach [8]

Im Simulationsmodell hat das PräkonzentratorMaterial einen Durchmesser von $800 \mu \mathrm{m}$ (die gesamte Hotplate) und eine Dicke von $50 \mathrm{~mm}$. In den später untersuchten Testmustern war die Hotplate allerdings nur zu einem kleinen Teil beschichtet, die beschichtete Fläche hat hier einen Durchmesser von ca. $300 \mu \mathrm{m}$. Wir gehen davon aus, dass dies im Wesentlichen zu einer Verringerung des Aufkonzentrationsfaktors führt und das Zeitverhaltens und die Vergleich zwischen Benzol und Toluol davon nicht beeinflusst wird. Zur Simulation der Funktion der Schicht wurde an den Grenzflächen ein Verteilkoeffizient definiert, der das Verhältnis der Gaskonzentration im Material zu dem in der Luft vorgibt. Durch schnelle Änderung dieses Parameters wird die Änderung der Temperatur des MOFs simuliert. Die Werte des Verteilkoeffizienten bei verschiedenen Temperaturen wurden aus den Ergebnissen der iGC-Messungen ermittelt [5].

\section{Ergebnisse der FEM-Simulationen}

Die Gaskonzentration in der Umgebungsluft betrug in den Simulationen $4 \cdot 10^{-7} \mu \mathrm{g} / \mathrm{m}^{3}$ was etwa $10 \mathrm{ppb}$ entspricht. Der Präkonzentrator war zunächst ausgeschaltet und adsorbiert das Gas aus dem Gehäuse und aus der Umgebungsluft. Der Zustand des Systems $6,5 \mathrm{~s}$ nach Start der ersten Adsorption ist in Abb. 3 a dargestellt. Die Gaskonzentration im Gehäuse ist stark reduziert, bis auf ca. $0,3 \mathrm{ppb}$ $\left(1,2 \cdot 10^{-8} \mu \mathrm{g} / \mathrm{m}^{3}\right.$, rechte Skala).

Im Präkonzentrator-Material ist insbesondere an der linken Ecke, an der der Durchgang zur Umgebung ist, die Konzentration stark angestiegen (linke Skala). 
a)

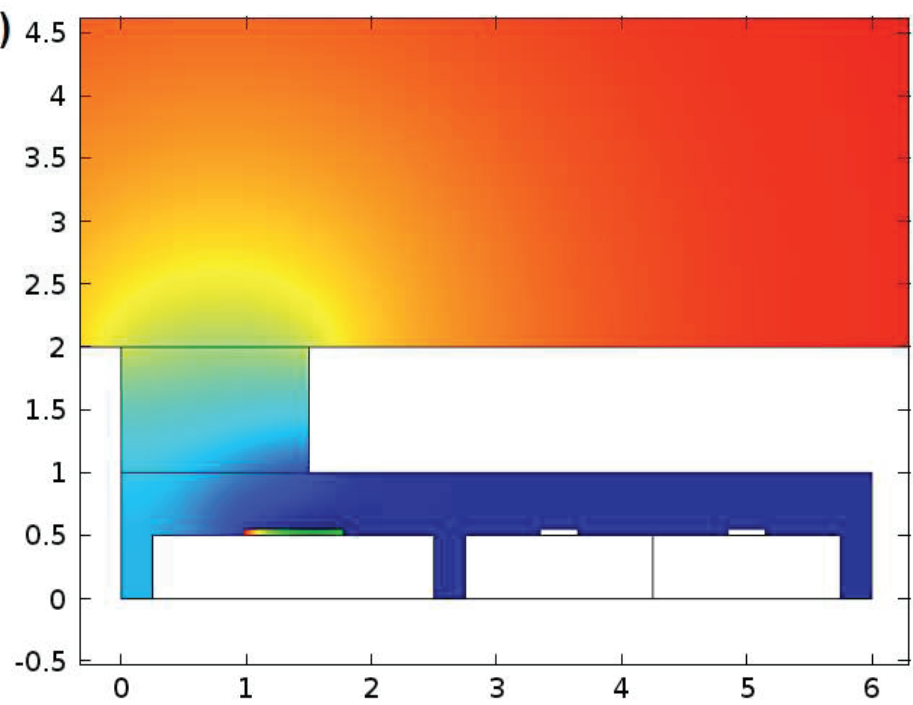

A $6.3291 \times 10^{-4}$ $\times 10^{-5}$

60

60

55

50

45

40

40

35

30

25

20

$1.9071 \times 10^{-4}$
$\Delta 4 \times 10^{-7}$

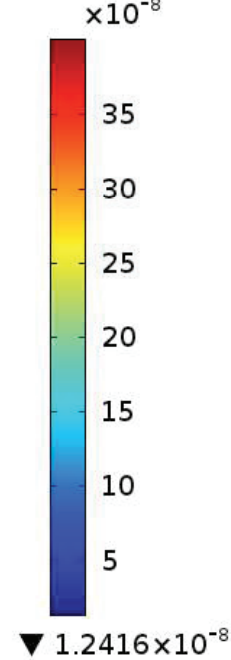

b)

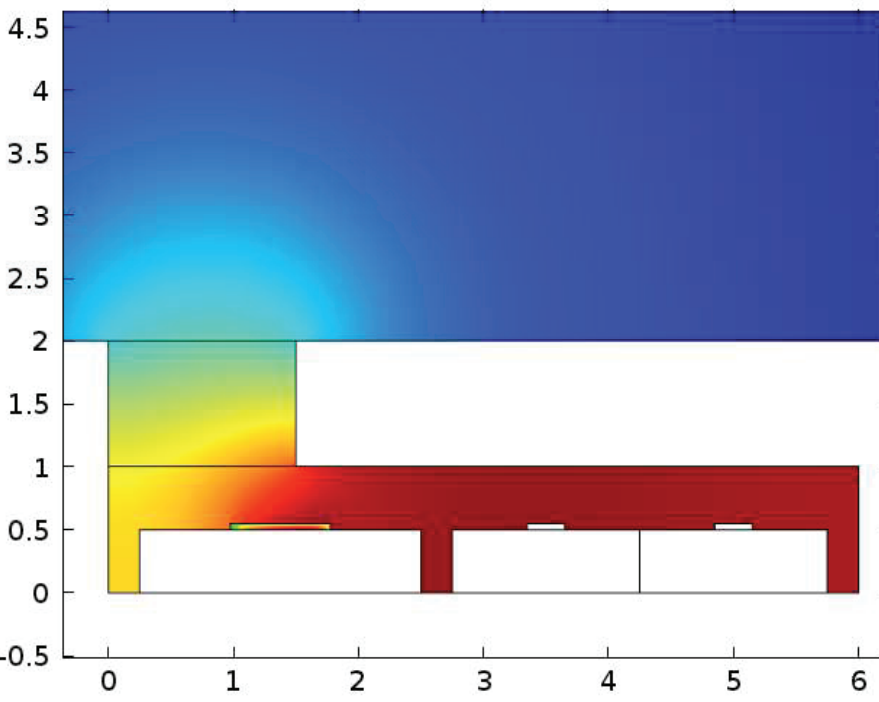

$\Delta 1.6122 \times 10^{-3}$

$\times 10^{-4}$

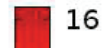

16

15

14

13

12

11

$9.8884 \times 10^{-4}$
$\Delta 1.1013 \times 10^{-5}$ $\times 10^{-6}$

10

8

6

4

2

Abb. 3: Verteilung der Gaskonzentration (in $\mu g / m^{3}$ ) im integrierten System zu verschiedenen Zeitpunkten einer Messung. a) $6.5 \mathrm{~s}$ nach Beginn der Gasadsorption; b) $1.5 \mathrm{~s}$ nach Beginn der Gasdesorption, nach [5]
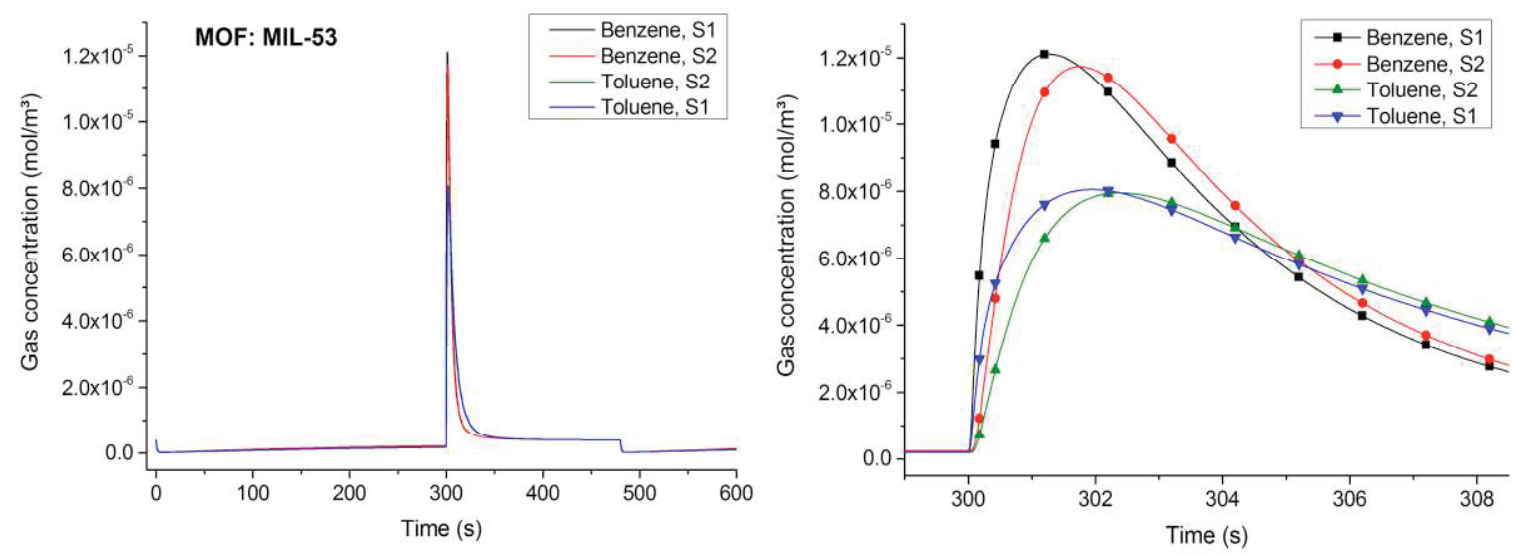

Abb. 4: Verlauf der Gaskonzentration an den zwei Gassensoren für Benzol und Toluol während eines 300 s Adsorptions- / Desorptions-Zyklus, nach [5] 
In der Simulation wurde nach $300 \mathrm{~s}$ der Verteilkoeffizient auf den Wert für $200^{\circ} \mathrm{C}$ umgeschaltet, das gesammelte Gas wird desorbiert. Abb. $3 \mathrm{~b}$ zeigt den Zustand 1,5 s nach Start der Desorption. Im Gehäuse ist die Gaskonzentration mit bis zu 275 ppb $\left(1,1 \cdot 10^{-5}\right.$ $\mu \mathrm{g} / \mathrm{m}^{3}$ ) gegenüber dem Ausgangswert stark erhöht, die höchste Konzentration findet sich im Bereich der zwei Sensoren.

Der zeitliche Verlauf der Gaskonzentration an den Sensoren ist in Abb. 4 dargestellt. Die Präkonzentrator-Temperatur während der Adsorptionsphase ist hier auf $50^{\circ} \mathrm{C}$ eingestellt. Man erkennt das ursprüngliche schnelle Abfallen der Konzentration zu Beginn, das langsame Ansteigen wenn der Präkonzentrator langsam gesättigt wird und den schnellen Desorptionspeak ab $300 \mathrm{~s}$. Bei $480 \mathrm{~s}$ wird der Verteilkoeffizient wieder umgeschaltet, und es kommt zu einer schnellen Readsorption des Gases im Gehäuse. Die Simulation wurde für zwei Zielgase, Benzol und Toluol, durchgeführt, und in Abb. 5 rechts sieht man deutlich dass die Konzentrationserhöhung für Benzol höher ist als die für Toluol. Die Faktoren, um die die Konzentrationen am Sensor S1 erhöht wurden sind 24,8 für Benzol und 16,6 für Toluol [5].

\section{Testmessungen und Ergebnisse}

Erste Testmessungen mit den Systemen wurden in trockener Luft durchgeführt, mit Benzol-Konzentrationen von $10 \mathrm{ppb}, 100 \mathrm{ppb}$ und $1000 \mathrm{ppb}$. Die zyklischen Betriebsmodi der Komponenten sind in Abb. 6 (links) abgebildet. Für die Adsorptionsphase (300 s) waren sowohl der Präkonzentrator als auch die Gassensoren ausgeschaltet. Nach 298 s wurden die Gassensoren zur Reinigung ihrer Oberflächen kurzzeitig auf $400{ }^{\circ} \mathrm{C}$ erhitzt. Zum Zeitpunkt $300 \mathrm{~s}$ im Zyklus wurden die Heizer der Sensoren dann auf $300{ }^{\circ} \mathrm{C}$ eingestellt und der Präkonzentrator-Heizer auf $200^{\circ} \mathrm{C}$ erhitzt. Nach einer Ausheizzeit von $180 \mathrm{~s}$ wurde der PC wider abgeschaltet, die Sensoren wurden bis zum Ende des Zyklus (600 s) auf ihrer Temperatur gehalten. Alle gezeigten Messkurven stammen vom Sensor S2 (siehe Abb. 2); S1 wird zunächst nicht betrachtet. Man sieht im Rohsignal (Abb 5 links), dass nach dem Umschalten der Sensortemperatur und der PC-Temperatur ein Peak im Signal entsteht, der jedoch auch ein generelles Sensorverhalten darstellt. Um sämtliche Einflüsse außer der Benzol-Desorption zu unterdrücken, wurden Sensorsignale normiert, indem Sensorsignal-Zyklen während der Benzol-Angebote punktweise durch Zyklen vor den jeweiligen Benzol-Angeboten dividiert wurden. Die Ergebnisse für die drei BenzolKonzentrationen sind in Abb. 5 rechts zu sehen.

Die Desorptionspeaks ab $300 \mathrm{~s}$ sind klar erkennbar und erreichen jeweils ca. den doppelten Wert des konstanten Signals nach der Desorption. Auffällig ist, dass der Peak des Zyklus in 10 ppb Benzol nahezu die Höhe des Peaks bei 100 ppb Benzol erreicht.

Insbesondere in dem normierten Signal von $1000 \mathrm{ppb}$ erkennt man weiterhin deutlich ein Einbrechen des Signals bei $480 \mathrm{~s}$, wenn der Präkonzentrator abgeschaltet wird. Dies ist eine Folge der schnellen Readsorption des Gases, vgl. Abb. 4 links.
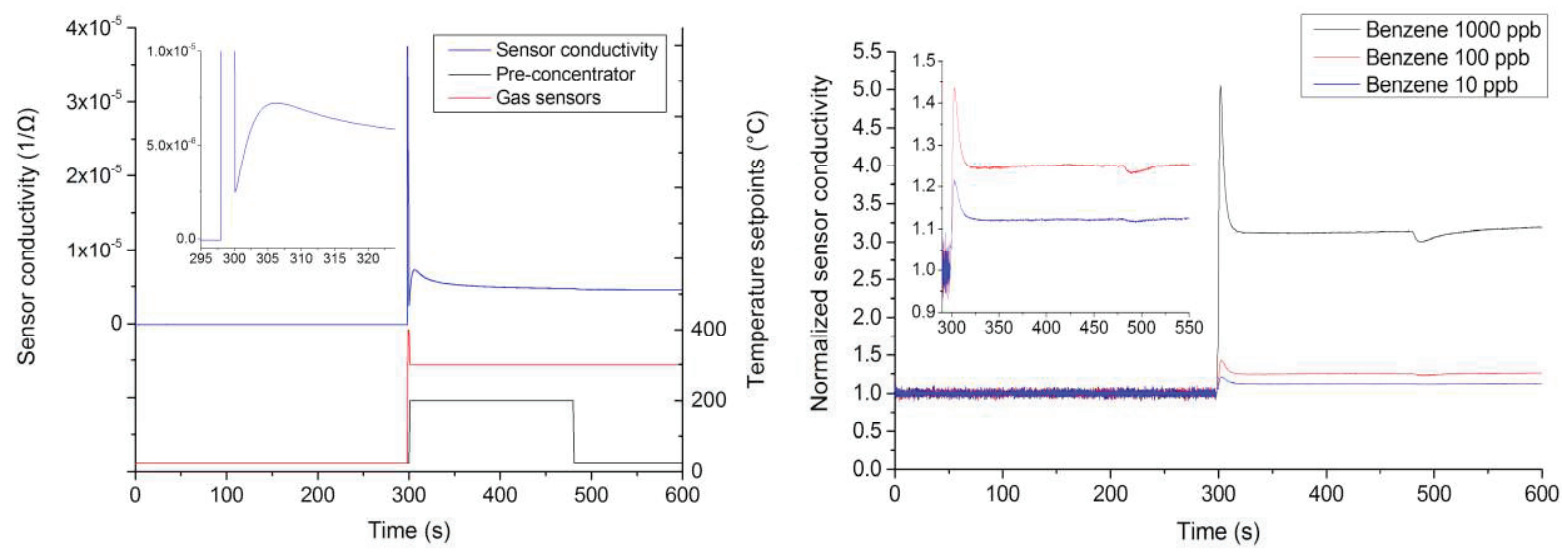

Abb. 5: Links: Temperaturverläufe der Komponenten des integrierten Systems (Gassensoren / Präkonzentrator) und Gassensor-Signal für 100 ppb Benzol; Rechts: Normierte GassensorSignale für die drei angebotenen Benzol-Konzentrationen [5,8] 

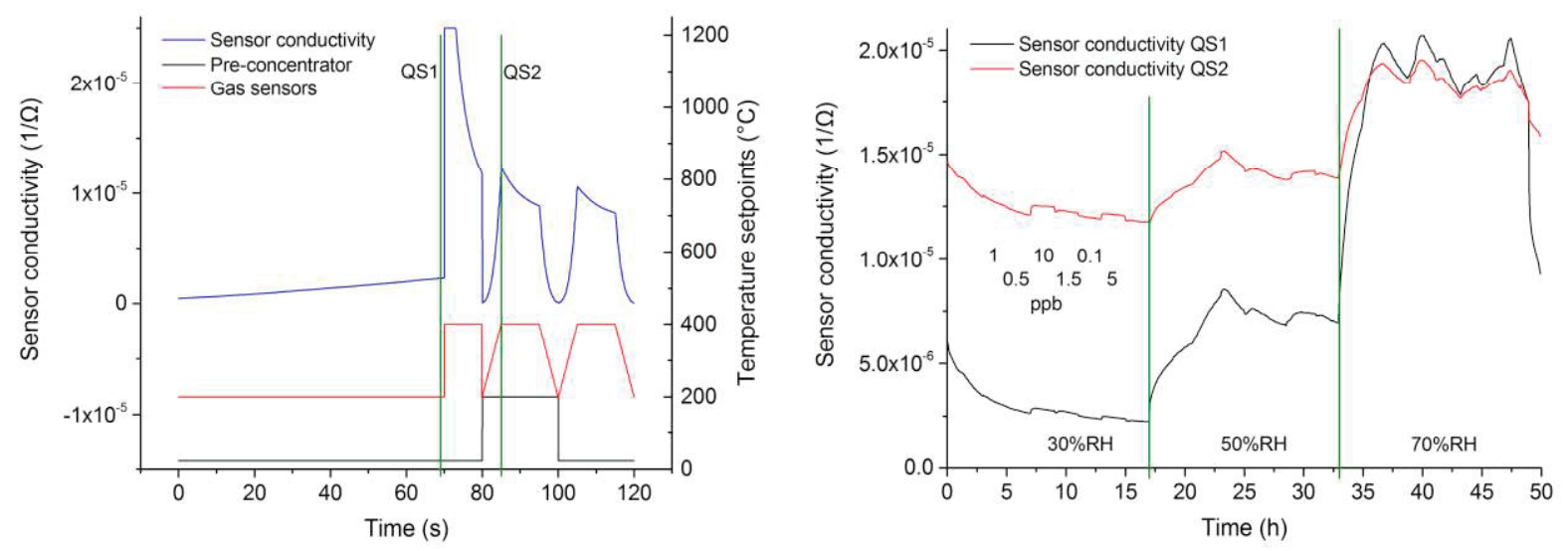

Abb. 6: Links: Temperaturverläufe der Komponenten des integrierten Systems (Gassensoren / Präkonzentrator) und Gassensor-Signal für 100 ppb Benzol; Rechts: Quasistatische Signale der Punkte QS1 und QS2 über den Verlauf der Messung bei verschiedenen Gasfeuchten

Betrachtet man die elektrische Leistung in diesem nicht optimierten Betriebsmodus, so erhält man eine durchschnittliche Leistungsaufnahme von $73,5 \mathrm{~mW}$ wenn beide Sensoren betrieben werden. Durch Verkürzung der Heiz-Zeiten ließe sich mit einem $600 \mathrm{~s}$ Zyklus die durchschnittliche Leistung auf $10,0 \mathrm{~mW}$ bei Betrieb mit zwei Sensoren und auf $5,7 \mathrm{~mW}$ bei Betrieb mit einem Sensor verringern [5].

Ausgehend von den positiven Ergebnissen wurden weitere Messungen mit niedrigen Benzol-Konzentrationen im Bereich des Akzeptanzwerts durchgeführt. Hierzu wurden sechs Benzolkonzentrationen in der Reihenfolge $1 \mathrm{ppb}, 0,5 \mathrm{ppb}, 10 \mathrm{ppb}, 1,5 \mathrm{ppb}$, $0,1 \mathrm{ppb}$ und $5 \mathrm{ppb}$ angeboten und diese wurde jeweils mit verschiedenen Luftfeuchtigkeiten gemessen (30\%RH, $50 \% \mathrm{RH}, 70 \% \mathrm{RH})$.

Weiterhin wurde ein komplexerer Temperaturzyklus für den Sensor genutzt (Abb. 6 links) Die genannten Konzentrationsprofile wurden jeweils einmal für jede Luftfeuchte angeboten.

Ausgewertet wurden hier zwei quasistatische Sensorsignale. Dazu wurden für zwei Punkte des Temperaturzyklus die Signale über den Verlauf der Messung aufgetragen, siehe Abb. 6 rechts. Die schwarze Kurve ist hier das Signal für einen speziellen Zeitpunkt im Zyklus und zwar am Ende des niedrigen Temperaturplateaus (siehe Markierung QS1), die rote Kurve das Signal nach dem Aufheizen des Sensors während des Ausheizens des Präkonzentrators (siehe Markierung QS2). Im Verlauf der Messung sieht man, dass für die geringe Feuchte klare Benzol-Signale während des Ausheizens des Präkonzentrators erkennbar sind (QS2). Bei Erhöhen der Feuchte sind die Benzol-Konzentrationen nicht mehr zu identifizieren. Auffällig ist der langsame Anstieg des Sensorsignals, was nicht an der Feuchtereaktion der Sensoren liegt. (Vergleichssensoren ohne Präkonzentrator im Sensorhousing zeigen diesen Anstieg nicht). Der Anstieg ist unabhängig von der Konzentration von Benzol. Wir vermuten, dass es sich bei diesem Effekt um eine Porenkondensation handelt und dass durch diesen Effekt konkurriende Adsorbate (z.B. Benzol) aus den Poren ausgetrieben werden, was den Anstieg des Sensorsignals bewirkt. Auch wenn es sich bei MIL-53 um ein hydrophobes Material handelt ist von einigen Gruppen Porenkondensation bereits bei geringer relativer Feuchte beschrieben worden [9].

In trockener Trägerluft ist somit ein klarer Desorptionspeak erkennbar, die Benzolkonzentration wird kurzzeitig deutlich erhöht. Bei Erhöhung der Feuchte wird dieser Effekt stark vermindert, vermutlich aufgrund von Porenkondensation von Wasser im MOF.

\section{Zusammenfassung und Ausblick}

Ein integriertes Präkonzentrator- GassensorMikrosystem zur Messung von Spurengasen wurde entwickelt, simuliert, integriert und getestet. Die Simulationen und die ersten Testmessungen zeigen die grundsätzliche Funktionalität des Konzepts, die Gaskonzentration an den Sensoren kann kurzzeitig deutlich erhöht werden.

Die Eignung des MIL-53 MOF-Materials zur Aufkonzentration von Benzol wurde mittels inverser Gaschromatographie gezeigt, die Desorptionseigenschaften konnten mittels Massenspektrometer ermittelt werden. Das Material zeigte gegenüber Tenax $®$ TA ein sehr viel höheres Adsorptionspotenzial. 
Mittels FEM-Simulationen konnten die Vorgänge innerhalb des Systems näher untersucht und das Design entsprechend optimiert werden. Hier konnte für Benzol eine Konzentrationserhöhung um den Faktor 28,8 erreicht werden.

Testmessungen in trockener Luft bestätigten die Eignung des Designs zur Detektion von Benzol in Luft. Trotz der gegenüber den Simulationen kleineren Mengen MOF konnten durch den Präkonzentrator die Sensorsignale um ca. einen Faktor 2 erhöht werden.

Messungen in feuchter Luft zeigten eine Problematik des PC-Materials auf, da hier vermutlich Porenadsorption von Wasser den Effekt des Präkonzentrators sehr stark verringert und das Gesamtsignal sogar verschlechtert.

Um diesem Problem entgegenzuwirken kann die Temperatur des MOFs in der Adsorptionsphase gegenüber der Raumtemperatur erhöht werden, dadurch sinkt die relative Feuchte. Da das MOF-Material bei $30 \% \mathrm{rH}$ offensichtlich noch keine Porenkondensation aufweist müsste eine moderate Erwärmung um $25^{\circ} \mathrm{C}$ über die Raumtemperatur, also z.B. auf $50^{\circ} \mathrm{C}$, ausreichen. Damit würde der Präkonzentrator durchgängig in einem Bereich betrieben werden, in dem keine Porenkondensation stattfindet. Weiterhin ist bei dieser Adsorptionstemperatur noch eine deutliche Anreicherung des Benzols zu erwarten wie die detaillierte Messung der Verteilkoeffizienten zeigt.

\section{Danksagung}

Dieses Projekt wurde gefördert durch das Siebte Rahmenprogramm der Europäischen Union für Forschung, Entwicklung und Demonstration unter Grant Agreement $\mathrm{Nr}$. 604311, Projekt SENSIndoor.

\section{Literaturnachweis}

[1] Geiss, O., Giannopoulos, G., Tirendi, S., Barrero-Moreno, J., Larsen, B. R., and Kotzias, $D$.: The AIRMEX study - VOC measurements in public buildings and schools/kindergartens in eleven European cities: Statistical analysis of the data, Atmos. Environ., 45, 3676-3684, doi:10.1016/j.atmosenv.2011.04.037, 2011.

[2] Schütze,A.: Integrated Sensor Systems for Indoor Applications: Ubiquitous Monitoring for Improved Health, Comfort and Safety, Procedia Engineering, 120, pp. 492-495, doi:10.1016/j.proeng.2015.08.681, 2015.

[3] European Parliament, Council of the European Union: Directive 2008/50/EC of the European
Parliament and of the Council of 21 May 2008 on ambient air quality and cleaner air for Europe, Official Journal of the European Union, Volume 51, 2008.

[4] Leidinger, M., Sauerwald, T., Reimringer, W., Ventura, G., and Schütze, A.: Selective detection of hazardous VOCs for indoor air quality applications using a virtual gas sensor array, J. Sens. Sens. Syst., 3 (2014), pp. 253263, doi:10.5194/jsss-3-253-2014.

[5] Leidinger, M., Rieger, M., Sauerwald, T., Alépée, C. and Schütze, A.: Integrated preconcentrator gas sensor microsystem for $\mathrm{ppb}$ level benzene detection, Sensors and Actuators $B$, submitted.

[6] Rowsell, J. L. C.; Yaghi, O. M. (2004). Metalorganic frameworks: a new class of porous materials. Microporous and Mesoporous Materials, 73, 3-14.

[7] Loiseau, T., Serre, C., Huguenard, C., Fink, G., Taulelle, F., Henry, M., Bataille, T., and Férey, G.: A rationale for the large breathing of the porous aluminum terephthalate (MIL-53) upon hydration, Chemistry 10, pp. 1373-1382, doi: 10.1002/chem.200305413, 2004.

[8] M. Leidinger, T. Sauerwald, M. Rieger, C. Alépée, A. Schütze: Integrated pre-concentrator gas sensor micro system for trace gas detection, Tagungsband Dresdner SensorSymposium, Dresden, 7.-9. Dezember 2015, doi: 10.5162/12dss2015/5.4, 2015.

[9] Canivet, J., Bonnefoy, J., Daniel, C., Legrand, A., Coasne, B., \& Farrusseng, D. (2014). Structure-property relationships of water adsorption in metal-organic frameworks. New Journal of Chemistry, 38(7), 3102-3111, DOI: 10.1039/C4NJ00076E. 